\title{
Structural transitions and complex domain structures across a ferroelectric-to-antiferroelectric phase boundary in epitaxial $\mathrm{Sm}-$ doped $\mathrm{BiFeO}_{3}$ thin films
}

\author{
C.-J. Cheng, ${ }^{1}$ D. Kan, ${ }^{2}$ S.-H. Lim, ${ }^{2}$ W. R. McKenzie, ${ }^{1}$ P. R. Munroe, ${ }^{1}$ L. G. Salamanca-Riba, ${ }^{2}$ R. L. Withers, ${ }^{3}$ I. Takeuchi, ${ }^{2}$ \\ and V. Nagarajan ${ }^{1}$ \\ ${ }^{1}$ School of Materials Science and Engineering, University of New South Wales, New South Wales 2052, Australia \\ ${ }^{2}$ Department of Materials Science and Engineering, University of Maryland, College Park, Maryland 20742, USA \\ ${ }^{3}$ Research School of Chemistry, Australian National University, Canberra, Australian Capital Territory 0200, Australia
}

(Received 19 December 2008; revised manuscript received 1 June 2009; published 21 July 2009)

\begin{abstract}
We have investigated structural phase transitions across a ferroelectric-to-antiferroelectric phase boundary in epitaxial (001) oriented $\mathrm{Bi}_{(1-\mathrm{x})} \mathrm{Sm}_{\mathrm{x}} \mathrm{FeO}_{3}$ thin films. For the $\mathrm{Sm}^{3+}$ concentration of $0.1 \leq x \leq 0.14$, we observe short-range antiparallel cation displacements, verified by the appearance of localized $\frac{1}{4}\{011\}$ weak reflections in the selected area electron diffraction patterns. At the critical composition of $x=0.14$, the system adopts a complex nanoscale domain mixture with appearance of $\frac{1}{4}\{011\}, \frac{1}{2}\{011\}, \frac{1}{2}\{010\}$, and $\frac{1}{2}\{111\}$ reflections and an incommensurate phase bridging the rhombohedral and orthorhombic phases. For compositions $0.14<x<0.2$, orientational twin domains coupled with antiphase oxygen octahedral tilts, identified by $\frac{1}{2}\{h k l\}$ weak superstructure are observed. The above systematic changes in the microstructure as a function of $\mathrm{Sm}^{3+}$ doping are linked to the macroscopic functional properties.
\end{abstract}

DOI: 10.1103/PhysRevB.80.014109

PACS number(s): 77.65.- j, 77.84.- $-\mathrm{s}$

\section{INTRODUCTION}

Structural origins play a central role in understanding the physical properties of ferroelectrics. From enhanced piezoelectric response in morphotropic phase boundary (MPB) ceramics ${ }^{1}$ to the role of microstructure in frustrated relaxors ${ }^{2}$ to antiferrodistortive phase transitions in $\mathrm{PbTiO}_{3}$ (Ref. 3) and $\mathrm{SrTiO}_{3},{ }^{4}$ the observed macroscopic ferroelectric (FE) properties have been closely linked to the interplay between the variation in underlying polar order and the corresponding crystal structure. In this work, we present a systematic study aimed at deciphering the subtle changes in local crystal structure and microstructure, and understanding how these changes give rise to various physical properties of the recently reported $\mathrm{Pb}$-free ferroelectric-antiferroelectric (AFE) phase boundary in $\mathrm{Sm}$-doped $\mathrm{BiFeO}_{3} .{ }^{5}$

$\mathrm{BiFeO}_{3}(\mathrm{BFO})$ has recently attracted enormous interest due to its multiferroic nature (i.e., co-existence of ferroelectricity and antiferromagnetism) as well as superior ferroelectric properties at room temperature. ${ }^{6}$ Although BFO has been identified as a possible environmentally friendly alternative to current $\mathrm{Pb}$-based piezoelectrics, it suffers from highleakage current, large coercive fields as well as possession of electromechanical coefficients much smaller than traditional $\mathrm{Pb}$-based compositions. In order to overcome the aforementioned shortcomings, $A$ - and/or $B$-site doping (of the BFO perovskite cell) has been attempted in order to improve both the electromechanical response as well as the leakage resistance..$^{7-9}$ The rationale behind such doping experiments is the fact that the lattice and, hence, functional behavior of a parent ferroelectric perovskite can be easily tuned through atomic substitution.

In particular, we recently reported on Sm-doped BFO $\left(\mathrm{Bi}_{(1-\mathrm{x})} \mathrm{Sm}_{\mathrm{x}} \mathrm{FeO}_{3}\right.$ or BSFO) epitaxial (001) thin films ${ }^{5}$ that exhibit a series of structural phase transitions as a function of $\mathrm{Sm}^{3+}$ doping, accompanied by concomitant changes in the functional properties. In particular, it was shown that the composition of $x=0.14$ represents a boundary between a rhombohedral and an (pseudo)orthorhombic, with the dielectric constant and the out-of-plane piezoelectric coefficient comparable to those of epitaxial (001)-oriented $\mathrm{Pb}\left(\mathrm{Zr}_{0.5} \mathrm{Ti}_{0.5}\right) \mathrm{O}_{3}$ (PZT) thin films. The properties of compositions on the (pseudo) orthorhombic side of the boundary are consistent with those which are typical of an AFE material. Thus, we refer to this material as the AFE phase.

In this work, we delineate the structural pathway, as well as the domain evolution, of the FE to AFE phase transition through the extensive use of electron diffraction analyses coupled with high-resolution transmission electron microscopy (HRTEM). Using the high sensitivity of electron diffraction to fine octahedral tilt transitions, systematic composition-dependent superstructure or weak reflections in electron diffraction patterns have been identified and carefully indexed by the pseudocubic unit-cell notation to match specific structural changes in the lattice. Such an approach has been previously implemented in a variety of lead and barium-based perovskites. ${ }^{10-15}$ Following Glazer's earlier approach, ${ }^{16,17}$ we demonstrate how BFO undergoes lattice changes to accommodate the rare-earth (RE) $A$-site $\left(\mathrm{Sm}^{3+}\right)$ dopant and as a result transforms to the antiferroelectric phase, first via antiparallel cation displacements and then eventually by antiphase oxygen octahedral tilts. In doing so, the system undergoes electromechanical softening yielding to complex nanodomains and ordering phenomena.

\section{EXPERIMENTAL DETAILS}

A series of epitaxial $\mathrm{Bi}_{(1-\mathrm{x})} \mathrm{Sm}_{\mathrm{x}} \mathrm{FeO}_{3}$ thin films with varying $\mathrm{Sm}$ compositions were fabricated on (001)-orientated $\mathrm{SrTiO}_{3}$ (STO) substrates with an epitaxial $\mathrm{SrRuO}_{3}$ (SRO) layer $(50 \mathrm{~nm})$ used as the bottom electrode using a pulsed laser deposition system (Pascal, Inc.). We use epitaxial thin films as it allows us to track precise changes in the crystal structure and, hence, clearly identify the resulting structure- 
property correlations. Care was taken to ensure that the thickness of the ferroelectric layer was kept constant for each composition. Details of the synthesis conditions $\left(\mathrm{O}_{2}\right.$ pressure, temperature, etc.) are given in Ref. 5. The composition of each film was confirmed with an electron probe microanalyzer (JEOL JXA 8900). Ferroelectric polarization hysteresis loops were obtained at $25 \mathrm{kHz}$ using a Radiant Premiere II loop analyzer. Capacitance vs voltage $(C-V)$ characteristics were measured with an HP 4275A LCR meter at a frequency of $100 \mathrm{kHz}$. Cross-sectional TEM specimens were prepared using an FEI Nova 200 Nanolab focused ion beam (FIB). This was done using the "lift-out" technique. ${ }^{18}$ Prior to the FIB processing, gold was sputtered onto the surface followed by platinum to protect the film surface during preparation. The electron transparent foils (approximately $15 \times 2.5 \mu \mathrm{m}^{2}$ ) were lifted out using a micromanipulator and placed onto carbon-coated copper mesh grid. For high-resolution imaging, plan-view specimens were prepared by conventional tripod-polishing procedures. The final thinning of the specimen was performed with argon ion milling on PIPS (Precision Ion Polishing System, Gatan, Inc.) with an accelerating voltage of $3.0 \mathrm{kV}$ to perforation. All images and electron diffraction patterns were recorded by JEOL 3000F TEM and CM200 TEM with the double-tilt holder. Reciprocal space map (RSM) was carried out using $\mathrm{x}$-ray four-circle diffractometer (Philips X'pert PRO MRD) with $\mathrm{Cu} K \alpha$ emission used to identify the crystallographic orientations. All patterns presented here are indexed by the pseudocubic unit-cell notation to match specific structural changes in the lattice. Bond-valence calculations were carried out using the program EUTAX and the bond valence parameters given in Refs. 19 and 20.

\section{RESULTS AND DISCUSSIONS}

We begin with a brief overview of the structure of BFO and the ramifications associated with replacing the $\mathrm{Bi}^{3+}$ ion with a smaller RE isovalent ion. At room temperature, BFO contains a network of corner-sharing oxygen octahedra, where $\mathrm{Fe}^{3+}$ cations are inside the octahedra and $\mathrm{Bi}^{3+}$ cations fill in between the cavities with $a^{-} a^{-} a^{-}$tilt system as per the Glazer notation ${ }^{16,17}$ which gives rise to a rhombohedrally distorted cell along the [111] pseudocubic direction. ${ }^{21,22}$ The relevant $\mathrm{RE}$ orthoferrite in this case, $\mathrm{SmFeO}_{3}$, has an orthorhombic structure with $a^{+} a^{-} a^{-}$mixed tilt system. ${ }^{23,24}$ Figure 1(a) is a schematic illustration of the smaller RE isovalent $\mathrm{Sm}^{3+}$ dopant ions substituting some of $\mathrm{Bi}^{3+}$ ions in the perovskite structure. According to the model proposed by Spaldin and co-workers, ${ }^{25}$ large ferroelectric order and spontaneous polarization in $\mathrm{BFO}$ primarily result from the stereochemically active $6 s^{2}$ lone pairs on the $\mathrm{Bi}^{3+}$ ions. Thus, systematic doping to replace the $\mathrm{Bi}^{3+}$ with smaller transitionmetal or RE dopants (in the present case $\mathrm{Sm}^{3+}$ ) may not only distort the cation spacing between the octahedra, which in turn could lead to rotations of the oxygen octahedra, but also alter the long-range ferroelectric order. The effect of the cation spacing on the stability of the perovskite cell can be understood semiquantitatively using the Goldschmidt tolerance factor $(t)$ framework, where systematic structural octa-
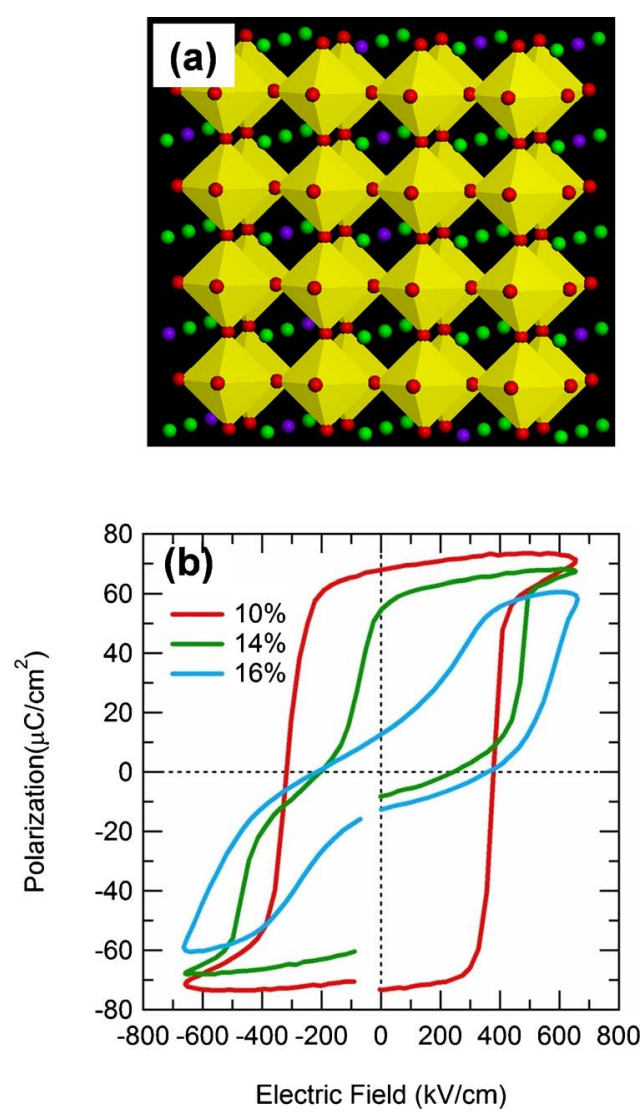

FIG. 1. (Color online) (a) Schematic illustration of $A$-site Smdoped $\mathrm{BiFeO}_{3}$ where the red spheres represent $\mathrm{O}^{2-}$ ions forming yellow octahedral cages, green spheres are $\mathrm{Bi}^{3+}$ ions, and purple $\mathrm{Sm}^{3+}$ ions have been randomly distributed and (b) experimental polarization hysteresis loops with $25 \mathrm{kHz}$ at compositions of $x$ $=0.1,0.14$, and 0.16 .

hedral tilt transitions may be expected. Reaney et al. ${ }^{11}$ used the tolerance factor approach to separate tilt transitions, where they showed that a value of $0.985<t<1.06$ gives an untilted structure, where as $0.964<t<0.985$ results in tilting in antiphase and $t<0.964$ results in both in-phase and antiphase tilting. For $\mathrm{Bi}_{(1-\mathrm{x})} \mathrm{Sm}_{\mathrm{x}} \mathrm{FeO}_{3}$ with $\mathrm{Bi}^{3+}(1.365 \AA)$ and $\mathrm{Sm}^{3+}(1.24 \AA)$, the tolerance factor $t$ as a function of $x$ was calculated to be less than 0.964 for all compositions in the range $0<x<0.4$, and thus, both in-phase and antiphase tilting of the oxygen octahedral are expected to occur.

These tilt transitions may induce significant changes in the ferroelectric phase behavior, and indeed this has been confirmed by a systematic change in the shape of the polarization hysteresis loop as a function of $\mathrm{Sm}^{3+}$ content. ${ }^{5}$ For an illustrative purpose, we plot typical hysteresis loops observed for $x=0.1$ (FE), 0.14 (FE-AFE phase boundary), and 0.16 (AFE) in Fig. 1(b). With increasing Sm, a slight distortion of the loop $(x=0.14)$ is seen which indicates the onset of AFE behavior. Finally, for compositions beyond $x \geq 0.14$ (in this case we show $x=0.16$ ), clear double loops are observed which has the characteristic of the AFE behavior.

We first present the results for a BFO thin film to show that effects reported in the Sm-doped films can be purely attributed to compositional changes, and not an artifact of 

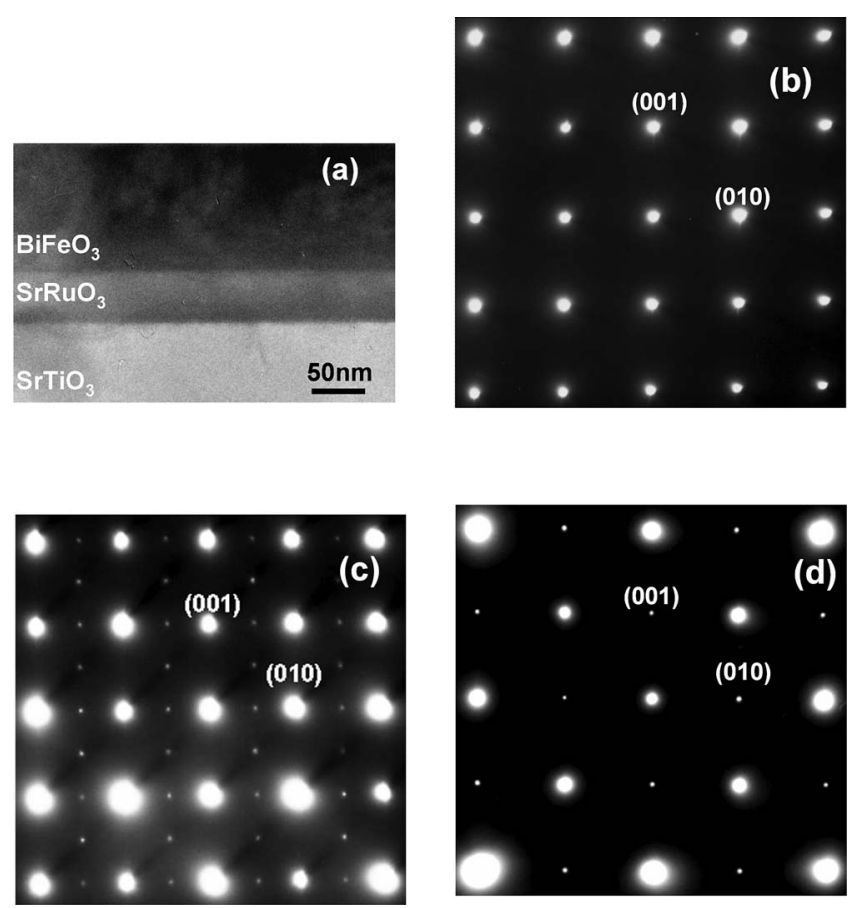

FIG. 2. (a) Cross-sectional TEM image of $\mathrm{BiFeO}_{3}$ films on (001)-orientated $\mathrm{SrTiO}_{3}$ substrate with $\mathrm{SrRuO}_{3}$ as bottom electrode; (b) $[100] \mathrm{ZADP}$ of $\mathrm{BiFeO}_{3}$; (c) [100] ZADP of $\mathrm{SrRuO}_{3}$; and (d) [100] ZADP of $\mathrm{SrTiO}_{3}$.

texturing due to an underlying substrate, i.e., distortions from either the presence of SRO or STO. Figure 2(a) shows the cross-sectional TEM image of a BFO thin film on a (STO) substrate with SRO as the bottom electrode. The image confirms that the BFO/SRO heterostructures with sharp interfaces and that there are no secondary phases developed during the thin-film deposition process. The [100] zone-axis selected-area diffraction patterns (ZADPs) of each layer are shown in Figs. 2(b)-2(d) for BFO, SRO, and STO, respectively. They agree very well with previous reports. ${ }^{6}$ The distinct differences between each pattern (for each respective layer) confirms that the chosen diffraction aperture in the TEM is sufficiently accurate to differentiate between each layer and thus provides credence for results and discussions hereafter that are wholly based on chemical effects (as opposed to substrate effects) for the BSFO films.

\section{A. Diffraction studies for the $\mathrm{Bi}_{0.9} \mathrm{Sm}_{0.1} \mathrm{FeO}_{3}$ (BSFO with $x$} $=0.1$ )

Figure 3(a) is a cross-sectional TEM image of the $\mathrm{Bi}_{0.9} \mathrm{Sm}_{0.1} \mathrm{FeO}_{3} / \mathrm{SRO} / \mathrm{STO}$ heterostructure. The inset to Fig. 3(a) is a ZADP from the bulk of the BSFO film showing the fundamental reflections along the [100] zone axis. However, we see that in local pockets that have a distinct contrast (shown by the arrow), in addition to the fundamental reflections, clear $\frac{1}{4}\{011\}$ superstructure reflections (circled) are present, as seen in Fig. 3(b). The corresponding HRTEM image acquired for such local regions is shown in Fig. 3(c). It displays distinct commensurate ordering along the [011] direction, and the optical diffraction pattern obtained via fast
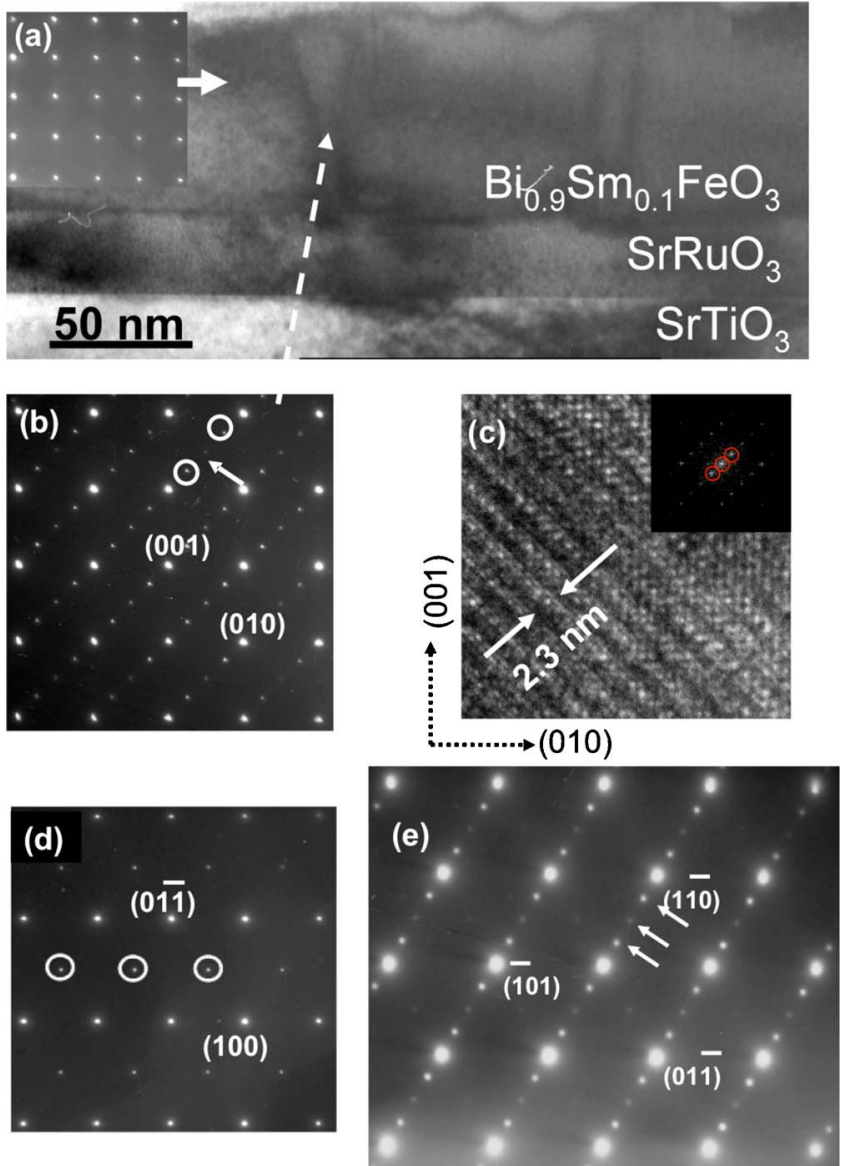

FIG. 3. (Color online) (a) Cross-sectional TEM image $x=0.1$ where inset shows only fundamental reflections; (b) [100] ZADP with circled $\frac{1}{4}\{011\}$ and arrowed $\frac{1}{2}\{011\}$ superstructure reflections from antiparallel cation displacement; (c) HRTEM image showing periodic ordering quadrupled along the (011) plane and the inset is corresponding FFT; (d) [011] ZADP showing circled $\frac{1}{2}\{11 \overline{1}\}$ reflections; and (e) [111] ZADP with arrowed superstructure reflections in good agreement with the [001] zone axis.

Fourier transform (FFT) (inset) is in close agreement with Fig. 3(b). The diffraction pattern presented in Fig. 3(b) shows a remarkable resemblance to $\langle 100\rangle$ ZADPs from the well-established antiferroelectric system of $\mathrm{PbZrO}_{3} \cdot{ }^{10,26} \mathrm{An}$ tiferroelectric phases arise from condensation of two phonon modes, i.e., condensation of the $\left(\frac{1}{2} \frac{1}{2} 0\right)(\pi / a) \Sigma_{3}$ and (110) $(\pi / \mathrm{a}) M_{5}$ which correspond to $\frac{1}{4}\{011\}$ and $\frac{1}{2}\{011\}$ reflections, respectively. ${ }^{27-29}$ Those softening modes give rise to antiparallel displacement of the $A$-site cations and result in quadrupling of the unit cell along the $\langle 011\rangle$ direction with dimensions of $\sqrt{2} a \times 2 \sqrt{2} a \times 2 a$, where $a$ is the lattice parameter of the pseudocubic perovskite unit cell. ${ }^{10,26}$ Indeed the ordering periodicity $(\sim 2.3 \mathrm{~nm})$ seen in Fig. 3(c) has a very close match with the above-suggested quadrupled structure. Figure 3(d) shows the corresponding [011] ZADP of the same sample, which also shows the appearance of $\frac{1}{2}\{011\}$ reflections. These so-called $\left\{h+\frac{1}{2}, k+\frac{1}{2}, l+\frac{1}{2}\right\}$ reflections or $F$ spots indicated by circles, are typically directly associated with antiphase tilting of oxygen octahedra. ${ }^{14,27,30,31}$ They are attributed to the antiphase rotations of the oxygen octahedra 
within the parent BFO lattice. It is critical to note that at $x$ $=0.1$, these superstructure patterns were observed only for some localized regions in the sample (typically 10-20 nm in size). In other regions, the weak spots are noticeably absent from the diffraction patterns, and instead have strong resemblance to the basic BFO pattern [as seen by comparing the inset to Figs. 2(b) and 3(a)]. This indicates that this composition is a two-phase mixture, where there are antiparallel cation displacement (most likely AFE) clusters in the fundamental FE matrix. Finally, Fig. 3(e) shows the [111] ZADP of the AFE clusters, where again it can be seen that similar to Figs. 3(b) and 3(c), the ordering is only along a single direction.

\section{B. Diffraction studies on $\mathrm{Bi}_{0.86} \mathrm{Sm}_{0.14} \mathrm{FeO}_{3}$ (BSFO with $\boldsymbol{x}=\mathbf{0 . 1 4}$ )}

As the $\mathrm{Sm}^{3+}$ content is increased further toward the composition of $x=0.14$, the diffraction data become highly complex. From our previous study, we expect that this composition resides at a FE-AFE phase boundary. Figure 4(a) is a typical the [100] ZADP that not only shows circled $\frac{1}{4}\{011\}$ and $\frac{1}{2}\{011\}$ reflections but also arrowed $\frac{1}{2}\{010\}$ reflections. It is to be noted though, that the intensity as well as the shape of these $\frac{1}{4}\{011\}$ spots are qualitatively different from those in the patterns shown in Fig. 3. Figure 4(b) shows the [111] ZADP of the same film. It is evident that in this case, the antiparallel cation shifts along the $\langle 011\rangle$ direction have become significantly suppressed as seen in relatively diminished $\frac{1}{4}\{011\}$ superstructure reflections, which is consistent with Fig. 4(a). Notably, the [011] ZADP [Fig. 4(c)] reveals that in addition to the appearance of circled $\frac{1}{2}\{011\}$ spots, there are incommensurate reflections along the [011] direction (arrowed satellite spots appear next to main reflections), which had not been observed previously in any of the prior RE doped studies of BFO. ${ }^{7}$

To understand the origins of this complex diffraction pattern, we performed detailed TEM investigations of the microstructure of this particular composition. We specifically discuss plan-view TEM results, as they provide a very clear perspective of the microstructural features and clearly reveal the mosaic domains in this system. Figure 5(a) is one such HRTEM plan-view micrograph that reveals the nanoscale phase mixture present in this composition. Optical diffraction (FFT) was employed to analyze the four principle representative regions (phases), and the corresponding patterns are shown in Fig. 5(b) through Fig. 5(e). First, at the top left corner of Fig. 5(a) (region I), it has only the fundamental perovskite reflections from the parent BFO lattice as indicated in Fig. 5(b). At the top right corner of Fig. 5(a) (region II), there is region of antiparallel cation displacements as evidenced by the pattern in Fig. 5(c), which is similar in structure to that of $\mathrm{Bi}_{0.9} \mathrm{Sm}_{0.1} \mathrm{FeO}_{3}$ discussed above in Sec. III A. At the bottom-left corner of Fig. 5(a) (region III), there are regions with the cell-doubled orthorhombic phase for which the FFT pattern is shown in Fig. 5(d). This structure is linked to the macroscopic AFE behavior (discussed in detail in Sec. III C). We have also looked at areas in between regions I, II, and III, and it was found that in the nanoscale regions on the right of Fig. 5(a), the FFT yields incommen-

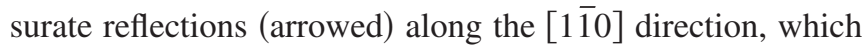
is marked as region IV [Fig. 5(e)]. Indeed, such fine-scale phase coexistence has been attributed to drastically improve functional response not only in ferroelectrics but also in magnetostrictive alloys. ${ }^{32,33}$

The $1 / x\{110\}$ incommensurate reflections [in Figs. 4(c) and $5(d)$ ] are similar to those seen in previously reported $\mathrm{Sn}$ or La substituted Zr-rich PZT solid solutions. ${ }^{14,34-36}$ Previously, incommensurately modulated structures have been broadly classfied as (i) displacively modulated, (ii) orientationally modulated, or (iii) interfacially modulated. In the case of the PLZT system, Viehland et al. ${ }^{37}$ have shown that the AFE PLZT has an orientational modulation, which also has a displacive component due to the electrostrictive strainpolarization coupling. Interfacially modulated incommensuration has been shown to arise from random faults at an interface between two commensurate regions. This phenomenon has been explained by Khachaturyan et al. ${ }^{38}$ with the aid of an adaptive phase martensite theory. A critical difference between interfacially modulated and displacive or orientational modulated incommensuration is that in the latter two cases, the positions of the $1 / x\{110\}$ incommensurate reflections with respect to the principal reflections do not change between different Brillouin zones. ${ }^{37}$ Furthermore, Khachaturyan et al. ${ }^{38}$ proposed that such incommensuration is possible when the scale of the structural heterogeneities is of the same order as the interplanar distance of the twinning plane.

Although it is difficult to quantitatively estimate the twinplane distance here, it is interesting to note that the incommensurate structure [seen in Fig. 5(e)] was found typically around regions of nanoscale structural inhomogeneity. Also in the case of Fig. 4(c) we measured subtle changes in the distance between the $1 / x\{110\}$ spots with varying Brillouin zones: this is strong indication that the incommensuration observed here is an interfacially modulated type. In the case of magnetic materials, incommensuration has been atrributed to competing interactions, where one set of nearest neighbors prefer an antiferromagnetic coupling while the next nearest set of neighbors prefer ferromagnetic coupling. It is perhaps not unreasonable that a similar scenario can be constructed here where incommensuration takes place not fortuitously but to adapt itself between antipolar AFE and the polar FE phases. That is, as described by Viehland et al. ${ }^{35,37,39}$ incommensurate structure could be caused by competition between softening in both zone-center (FE) and zone-boundary (AFE) phonon modes at $x=0.14$. This is also deduced again from the observation that the incommensurate phase typically occurs spatially between the parent FE and the phase separated AFE regions (Fig. 5). Thus, we propose that it is a bridging phase, i.e., a compromise between the FE and AFE states. Both an adaptive like phase and incommensuration are often associated with improved electromechanical properties $^{28,35,40,41}$ and this could indeed explain why the composition at $x=0.14$ was previously found to have enhanced piezoelectric and dielectric properties.

We further need to explain the presence of both the $\frac{1}{2}\{010\}$ and $\frac{1}{2}\{011\}$ reflections in Fig. 4(a) that were distinctly absent in the previous $\mathrm{BFO}$ and $\mathrm{Bi}_{0.9} \mathrm{Sm}_{0.1} \mathrm{FeO}_{3}$ films. Al- 

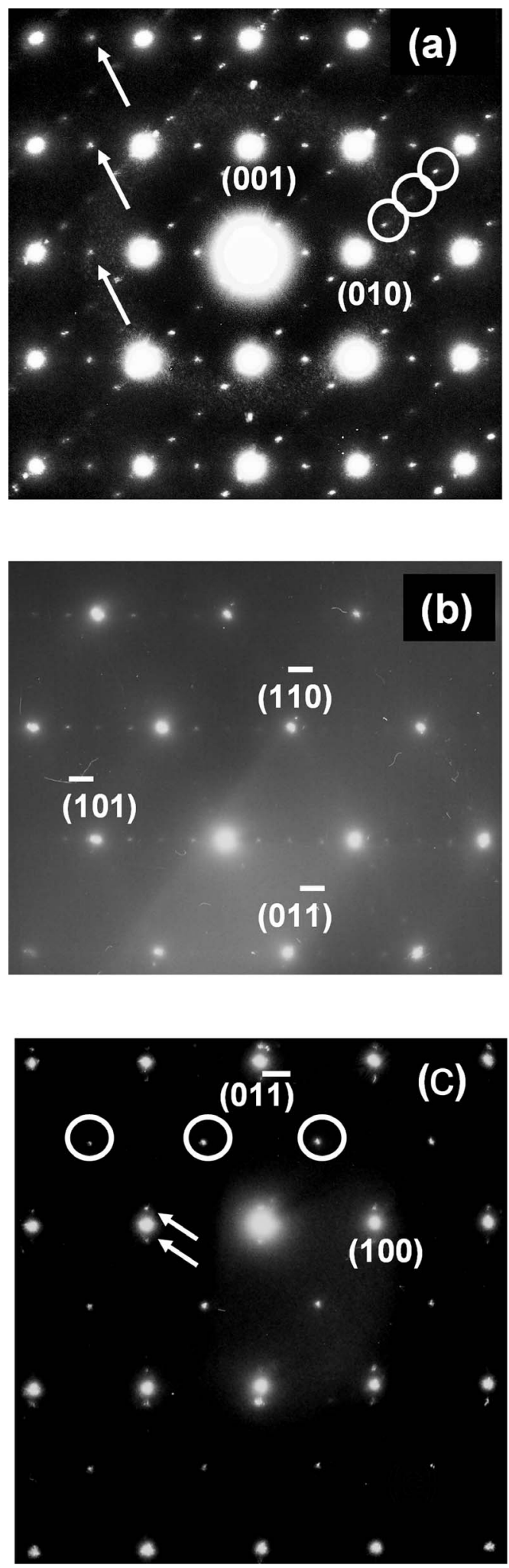

FIG. 4. Diffraction pattern of $x=0.14$ (a) [100] ZADP showing both circled $\frac{1}{4}\{011\}$ and $\frac{1}{2}\{011\}$ superstructure reflections and arrowed $\frac{1}{2}\{010\}$ reflections, suggesting the FE and AFE phase transitions are occurring; (b) [111] ZADP with arrowed superstructure has become much weaker in localized regions; and (c) [011] ZADP with circled $\frac{1}{2}\{11 \overline{1}\}$ reflections as well as arrowed incommensurate ordering. (a)
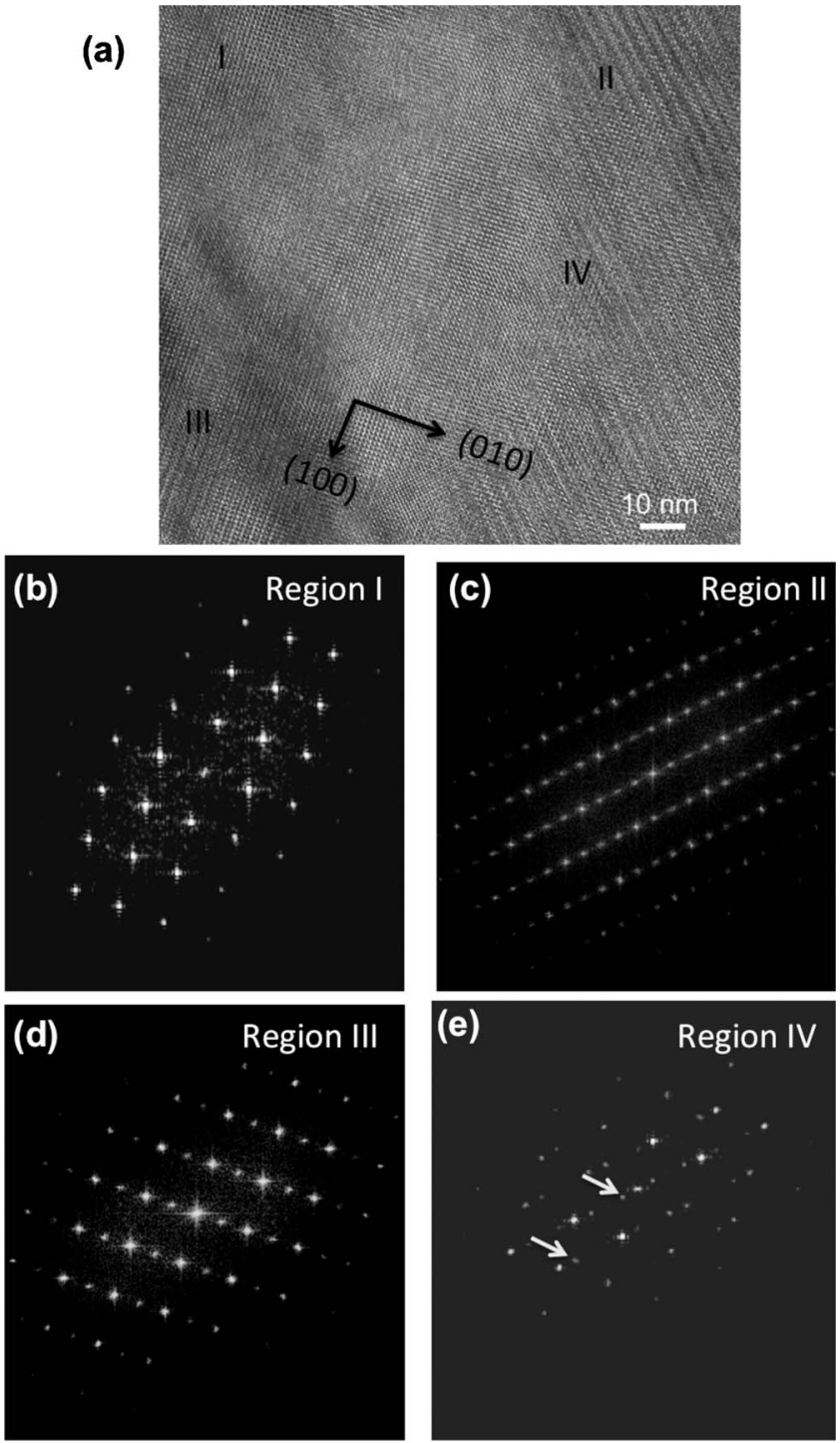

(e)

Region IV

FIG. 5. (a) Plan-view zoom in HRTEM image of $x=0.14$ indicating complex nanoscale mixture consisting of region I, region II region III, and region IV, (b) FFT of region I with fundamental reflections; (c) FFT of region II with cell quadrupled antiparallel cation shift reflections; (d) FFT of region III with cell doubled orthorhombic reflections; and (e) FFT of region IV with incommensurate reflections.

though it is tempting to attribute these reflections merely to the underlying SRO layer, [see Fig. 2(c)] it must be recalled that these reflections were not present either in the case of the BFO or the $x=0.1$ films, which were of the similar thickness. On the contrary, it will be shown in Sec. III C that the $\frac{1}{2}\{010\}$ reflections are specifically due to $\mathrm{Sm}^{3+}$ doping, which induces a cell-doubling phase transition along the $b$ axis of an (pseudo) orthorhombic structure, whereas the $\frac{1}{2}\{011\}$ spots are due to structural orthogonal twins (or variants).

Figure 6 is a plan-view HRTEM image of the same $x$ $=0.14$ sample focused on a region which has a mixture of the AFE regions with patterns corresponding to Figs. 5(b) and 5(c). The corresponding selected-area electron diffraction (SAED) is shown as an inset where spots corresponding to both, $\frac{1}{4}$ and $\frac{1}{2}$ orderings, are visible. The micrograph also 


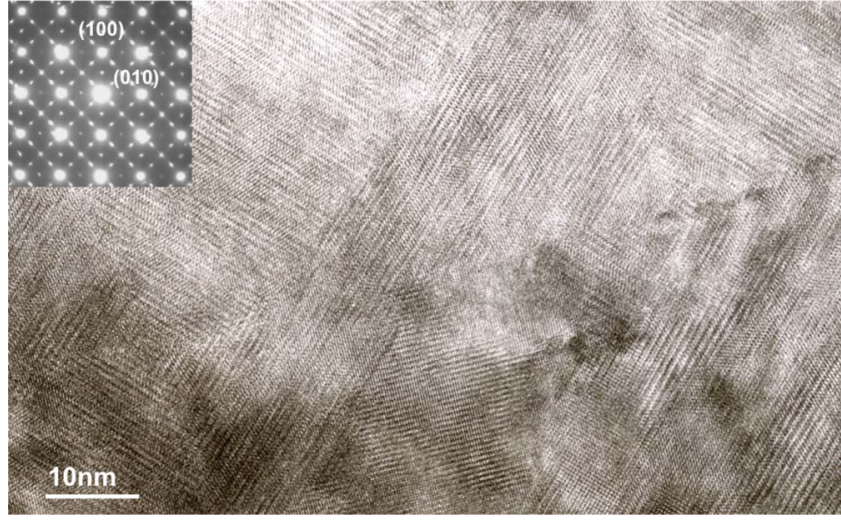

FIG. 6. (Color online) Plan-view HRTEM image of $x=0.14$. The nanodomains $20-50 \mathrm{~nm}$ in size observed with twin boundaries. The electron diffraction of this region is shown in the inset with superposition of nanodomains arranged approximately $90^{\circ}$.

shows the presence of a large density of twins. There is approximately $90^{\circ}$ rotation between [100] and [010] orientations of the twins, which is also reflected in the SAED. A similar nanotwin structure has recently been observed at the vicinity of other MPB piezoceramics. ${ }^{42}$ The size of the domains with same twin orientations are around $20-50 \mathrm{~nm}$, which is quite small and can be considered as a result of the minimization of the domain-wall energy at $x=0.14$. Such nanodomains have been previously associated with the outstanding piezoelectric response since low domain-wall energies with a smaller domain structure can be perturbed more easily by electric field. ${ }^{43-47}$ More recently, small domains have also been observed by piezoelectric force microscopy in lanthanum-doped $\mathrm{BiFeO}_{3}$, where a relatively high domainwall density leads to reduction in electric field in BFO. ${ }^{7}$ Thus, one finds that at $x=0.14, \mathrm{Sm}^{3+}$ doping brings about highly intricate structural changes to the original simple perovskite lattice framework.

\section{Diffraction studies on $\mathrm{Bi}_{0.8} \mathrm{Sm}_{0.2} \mathrm{FeO}_{3}$ (BSFO with $x=0.2$ )}

When more $\mathrm{Sm}$ is added beyond $x=0.14$ (i.e., for the $x$ $=0.2$ composition), the antiparallel cation displacement component totally vanishes, as signified by the disappearance of the $\frac{1}{4}\{011\}$ spots. Instead, one finds strengthening of two other distinctive superstructure spots highlighted by the arrowed and boxed positions of $\frac{1}{2}\{010\}$ and $\frac{1}{2}\{011\}$, respectively, as shown in Fig. 7(a). It is important to note that patterns such as the one in Fig. 7(a) are observed in all parts of the $\mathrm{Bi}_{0.8} \mathrm{Sm}_{0.2} \mathrm{FeO}_{3}$ sample, implying that this is not a cluster effect. Furthermore, higher order reflections show peak splitting (circled), which is an indication of possible existence of fine twin structures (similar to the ones seen in Fig. 6) with a twin boundary along the (110) plane. ${ }^{48}$

Figure 7(b) is a cross-sectional view of this sample showing an upper and lower region separated by a (domain) boundary as indicated by the dashed line. As illustrated in the inset diffraction patterns, two distinctive types of weak reflections at the positions of $\frac{1}{2}\{010\}$ (circled-lower inset) and $\frac{1}{2}\{011\}$ (circled-upper inset) are visible. We find that the
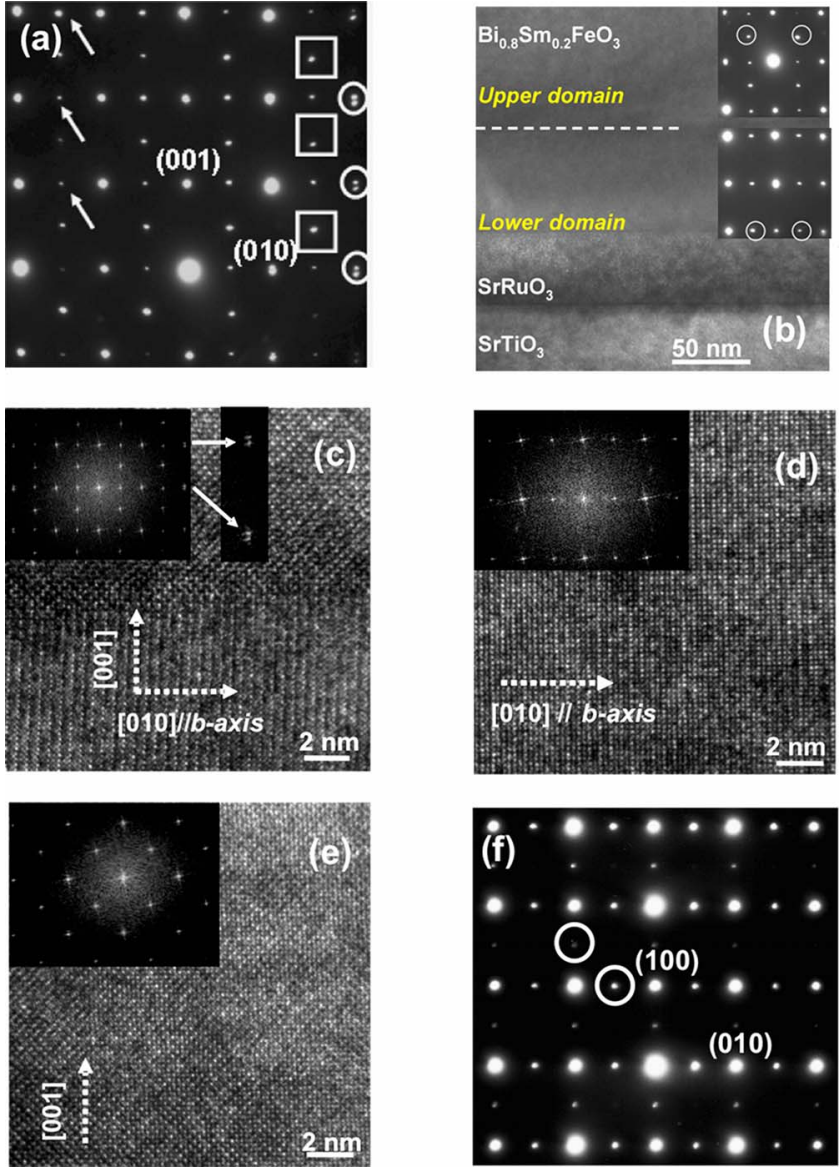

FIG. 7. (Color online) (a) The [100] ZADP of $x=0.2$; (b) crosssectional TEM image with two distinct domains separated by a domain wall indicated by the dashed line. Lower insets displays weak $\frac{1}{2}\{010\}$ reflections, whereas in the upper inset $\frac{1}{2}\{011\}$ reflections are present; (c) HRTEM image showing domain boundary with the corresponding FFT shown as inset. The arrows in the inset point to zoomed higher order reflections with peak splitting as an indication of twinning; (d) lower domain with corresponding FFT inset showing $\frac{1}{2}\{010\}$ reflections with a cell-doubled ordering along the $b$ axis; (e) HRTEM image of the upper domain with FFT inset showing fundamental reflections rotated by $45^{\circ}$ along the [001] direction; and (f) shows the corresponding plan-view diffraction pattern with $\frac{1}{2}\{010\}$ and $\frac{1}{2}\{100\}$ weak reflections in addition to fundamental spots.

$\frac{1}{2}\{010\}$ reflections become weaker as one moves away from the bottom along the [001] direction, whereas $\frac{1}{2}\{011\}$ reflections become more prominent. This suggests that the origins of these two types of superstructure spots are different (and not from the SRO layer). The high-resolution TEM image in Fig. 7(c) taken across the domain boundary reveals a double periodicity in the lower domain that is not present in the upper domain. (In the orthorhombic notation, this is along the $b$ axis.) For clarity, the lower and upper domains are shown separately in Figs. 7(d) and 7(e), respectively, with their FFTs (shown in insets). The lower domain, shown in Fig. 7(d), has a repetitive ordered structure with doubling of periodicity approximately every $0.8 \mathrm{~nm}$, i.e., along the orthorhombic $b$ axis (arrow direction) which would result in $\frac{1}{2}\{010\}$ reflections. This is verified by the FFT patterns of the 


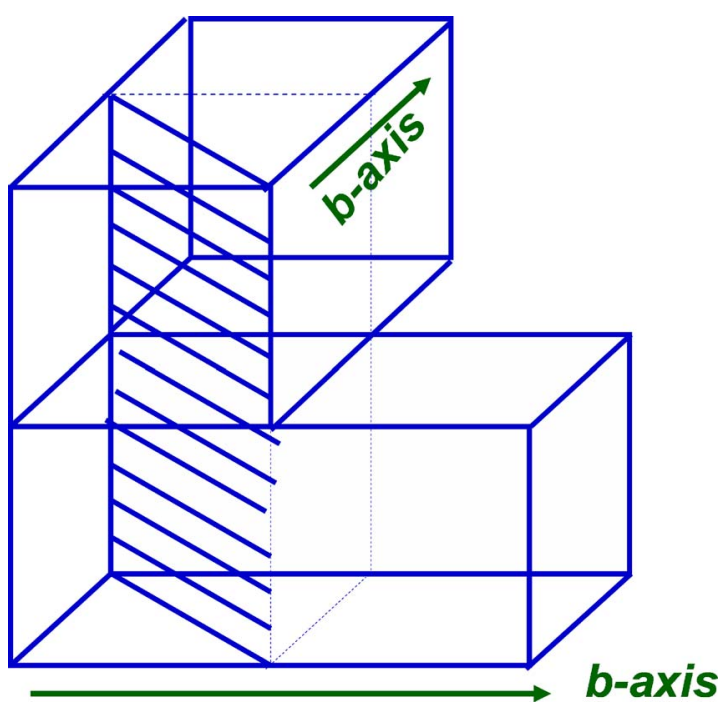

FIG. 8. (Color online) Graphical illustration of the orientational variants showing upper domain with $b$ axis at $90^{\circ}$ to the bottom domain. In the upper domain the $b$ axis is normal to the plane of the page while in the lower domain it is along the direction of the arrow.

lower region that show extra spots at $\frac{1}{2}$ positions along the $b$ axis. The FFT analysis of the upper domains [inset in Fig. 7(d)] shows fundamental perovskite reflections albeit at nearly $45^{\circ}$ to the direction along the cell-doubled $b$ axis of Fig. 7(d). Superimposing the two FFT patterns in Figs. 7(d) and 7(e), one can obtain the FFT in the inset of Fig. 7(c), which is also identical to the diffraction patterns in Figs. 7(a) and 7(b). The FFT pattern also confirms that the splitting of the spots is due to the overlap from these domains as shown in the enlarged inset in Fig. $7(\mathrm{c})$. Thus, the $\frac{1}{2}\{011\}$ reflections in [100] ZADP come from the twin variants rather than lattice-related tilt transitions. This is further confirmed by a corresponding plan-view diffraction pattern viewed down the [001] zone axis [Fig. 7(f)]. It shows main perovskite reflections along with circled $\frac{1}{2}\{010\}$ and $\frac{1}{2}\{100\}$ spots, i.e., absence of weak $\frac{1}{2}\{011\}$ reflections. This indicates the upper domain in Figs. 7(c) and 7(d) is an approximately $90^{\circ}$ rotation of the lower domain with a (110) mirror plane where in the upper domain, the $b$ axis is normal to the plane of the page, while in the lower domain it is along the direction of the arrow. The schematic of this complex twin structure is graphically illustrated in Fig. 8 with the hashed mirror plane.

Finally, we present diffraction analyses conducted to investigate the origin of the AFE mechanism at $x=0.2$. Thus far, two possible reasons have been proposed for the origin of AFE behavior-antiphase rotation of oxygen octahedra ${ }^{17}$ and antiparallel cation displacements. ${ }^{49}$ Additionally, one cannot ignore the possibility of chemical ordering of cations $^{50,51}$ to induce changes to the shape of the polarization loop. First, the concentration ratio between $\mathrm{Bi}^{3+}$ and $\mathrm{Sm}^{3+}$ cations is not 1:1. Second, we have confirmed via bondvalence calculations ${ }^{19,20}$ that the bond-valence sum parameter $R_{0}\left(\mathrm{Sm}^{3+}-\mathrm{O}\right)=2.088 \AA$ is virtually identical to $R_{0}\left(\mathrm{Bi}^{3+}-\mathrm{O}\right)=2.090 \AA$, and hence there is clearly very little driving force for $\mathrm{Bi}^{3+} / \mathrm{Sm}^{3+}$ ordering from the pure bond-
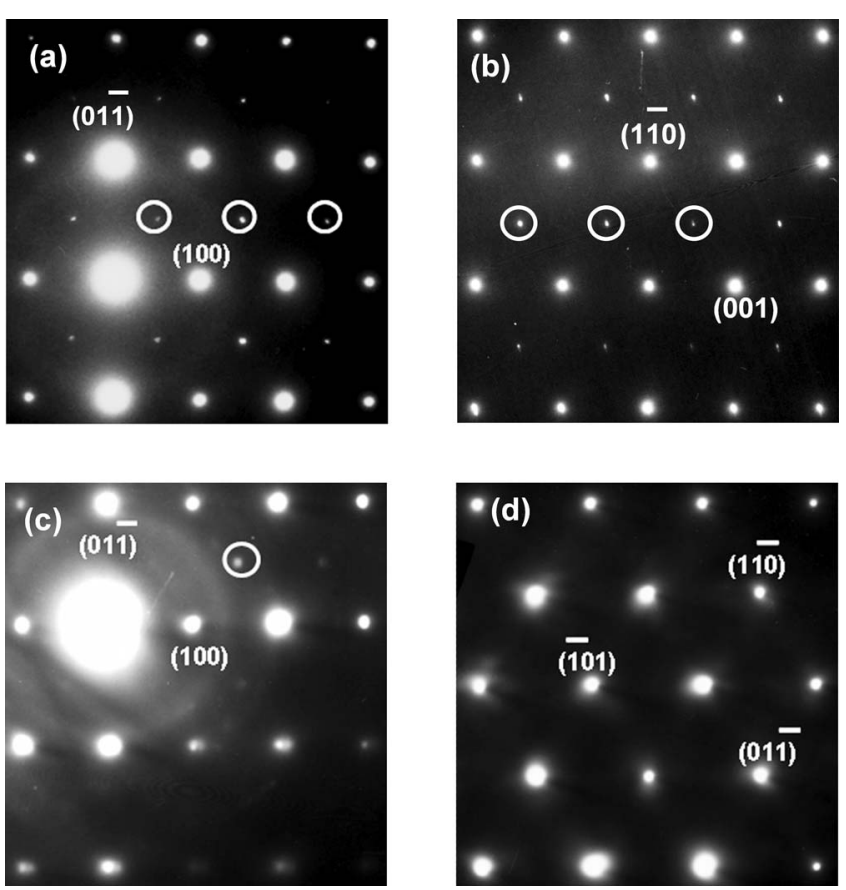

FIG. 9. (a) The [011] ZADP from $x=0.2$ sample showing $\frac{1}{2}\{11 \overline{1}\}$ reflections related with antiphase oxygen octahedral tilts are indicated by circles; (b) [110] ZADP with $\frac{1}{2}\{1 \overline{1} 1\}$ reflection confirming cell doubling takes place along both variants; (c) [011] ZADP by tilting toward $(3,1, \overline{1})$ spot with $\left(\frac{3}{2}, \frac{1}{2}, \frac{1}{2}\right)$ remain standing and $\left(\frac{1}{2}, \frac{1}{2}, \frac{1}{2}\right)$ reflections have already vanished; and (d) [111] ZADP with fundamental spots only.

valence sum point of view alone. As this composition has an orthorhombic structure with unit-cell dimensions close to $\mathrm{SmFeO}_{3}$, bond lengths from the parent $\mathrm{SmFeO}_{3}$ orthoferrite $^{20}$ were used as an approximation. For example, using the cell dimensions and coordinates given in the reported Pnma crystal structure of $\mathrm{SmFeO}_{3}$ (Maslen et al. ${ }^{20}$ ), the calculated valence $V_{\mathrm{i}}=\Sigma_{\mathrm{i}, \mathrm{j}} S_{\mathrm{i}, \mathrm{j}}$ gives $V(\mathrm{Sm})=3.011$, $V(\mathrm{Fe})=3.017, V(\mathrm{O} 1)=2.122$, and $V(\mathrm{O} 2)=1.953$. Using the same coordinates but substituting $\mathrm{Bi}$ for $\mathrm{Sm}$, the equivalent valences are virtually identical, i.e., $V(\mathrm{Bi})=3.027, V(\mathrm{Fe})$ $=3.017, V(\mathrm{O} 1)=2.128$, and $V(\mathrm{O} 2)=1.958$. Therefore, chemical cation ordering is highly unlikely (also confirmed by elemental mapping analysis). In order to differentiate between rotation of oxygen octahedra and antiparallel cation displacement, we performed systematic ZADP investigations around the [011] and [110] zone axis for the $x=0.2$ shown in Figs. 9(a) and 9(b), respectively. The pattern has distinct $\left\{h+\frac{1}{2}, k\right.$ $\left.+\frac{1}{2}, l+\frac{1}{2}\right\}$ reflections or $F$ spots indicated by the circles. It should be noted that in electron diffraction, dynamical, rather than perfect kinematical, scattering occurs and hence multiple scattering is almost inevitable, and this affects the diffraction patterns. Therefore, this provides explanation for any for double diffraction that may exist which is best explained by a model put forth by Reaney et al. ${ }^{11,30,52}$ who have considered the scheme of the $\langle 110\rangle$ ZADP where the double diffraction route, namely, $\left(\frac{3}{2}, \frac{1}{2}, \frac{1}{2}\right)+(\overline{1}, 0,0)$ leads to the appearance of the $\left(\frac{1}{2}, \frac{1}{2}, \frac{1}{2}\right)$ reflection as in the present case [as seen in Figs. 9(a) and 9(b)]. Indeed, such a spot 
where $h=k$ is kinematically forbidden in the Glazer analysis. Since we have used model epitaxial films, it is possible to verify if the double-diffraction model described above holds true.

Figure 9(c) best demonstrates this, where the electron beam is tilted toward $(3,1, \overline{1})$ around $3-4$ degrees from the [011] zone axis. Now the pattern shows a much stronger $\left(\frac{3}{2}, \frac{1}{2}, \frac{1}{2}\right)$ reflection, but more importantly, the $\left(\frac{1}{2}, \frac{1}{2}, \frac{1}{2}\right)$ reflections have now disappeared. In fact, close inspection of the pattern in Fig. 9(c) reveals that all weak reflections, where $h=k$, have disappeared in accordance with Glazer's conditions for antiphase oxygen octahedral tilts. Furthermore, from Ricote et al. ${ }^{31}$ we know that antiparallel cation displacement would yield $\frac{1}{2}\{h k 0\}$ reflections in the $\langle 110\rangle$ ZADP. These are, however, clearly absent in Figs. 9(a) and 9(b). Hence, this explanation can be precluded. ${ }^{10,30}$ This was also confirmed by the [111] ZADP shown in Fig. 9(d) which does not show any $\frac{1}{2}\{h k 0\}$ spots. Thus, one concludes that $\{h$ $\left.+\frac{1}{2}, k+\frac{1}{2}, l+\frac{1}{2}\right\}$ spots are due to the effect of oxygen octahedra with antiphase tilting. ${ }^{12,14,27,30,31}$ We propose that rotation in one octahedron around the tilt axis would cause an antiparallel rotation in all neighboring octahedra around that axis. ${ }^{17,53}$ This results in doubling of the repeat distances perpendicular to the tilt axis ${ }^{16,17}$ and subsequent presence of $\frac{1}{2}\{h k l\}$ spots in the diffraction patterns.

The presence of antiphase tilts would also result in the relevant lattice vectors not being normal, but inclined to each other ${ }^{17}$ due to the distortions of the octahedra. This would lead to a difference in the $d$ spacing and the Bragg angle in the $\omega-2 \theta$ space and ultimately splitting of the spots in the same $\{h k l\}$ family in asymmetric RSM. ${ }^{54}$ This is confirmed in Fig. 10(a), which is a RSM measured with an X-ray diffractometer (Philips MRD) around the fundamental (211) reflections for a 200-nm-thick (001)-oriented epitaxial film of $\mathrm{Bi}_{0.8} \mathrm{Sm}_{0.2} \mathrm{FeO}_{3}$. The film does display splitting of the (211) and $(21 \overline{1})$ reflections with the right-most spot identified as the substrate (211) peak. The corresponding cross-sectional $2 \theta$ line scan is shown in Fig. 10(b). CaRIne Crystallography 3.1 was used to find the best fit, obtained for a structure with fundamental (perovskite) lattice parameters $a=3.87 \AA, b$ $=3.895 \AA$, and $c=3.915 \AA$ and $\alpha=89^{\circ}, \beta=89.2^{\circ}$, and $\gamma$ $=89.9^{\circ}$. The numerical simulations using these parameters confirm that the peaks are $(211)$ and $(21 \overline{1})$ reflections with both the experimental $(57.19,58.33)$ and simulated $2 \theta$ values $(57.2,58.34)$ closely agreeing with each other.

To illustrate the critical role of the $\frac{1}{2}\{h k l\}$ in the observed AFE behavior and see if their disappearance leads to a concomitant loss of AFE behavior, we focused on the temperature dependence of the BSFO thin film. Although it was possible to extract the temperature dependence of the intensity of the $\frac{1}{2}\{h k l\}$ spots for the AFE side of the composition space, we found that the temperatures at which they began to disappear were quite high $\left(\sim 400{ }^{\circ} \mathrm{C}\right)$. At these temperatures it was not possible to get stable polarization hysteresis loops without prominent leakage and furthermore the films suffered from significant $\mathrm{Bi}$ loss. Thus we resorted to an alternative approach, where we demonstrate that the appearance of the $\frac{1}{2}(\{h k l\}$ spots can be linked to onset of antiferroelectricity. (a)

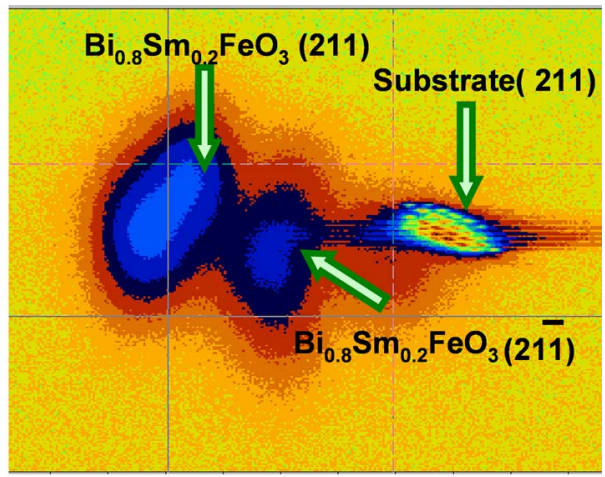

(b)

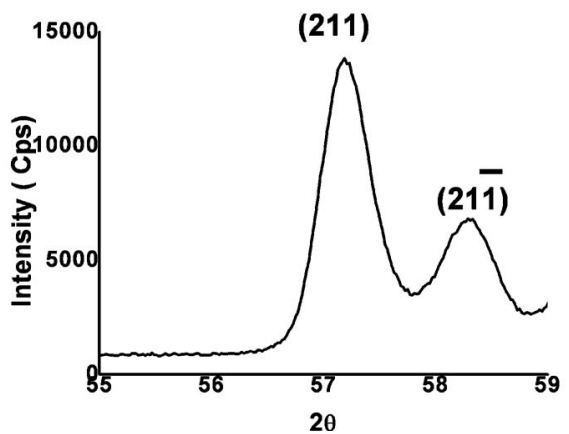

FIG. 10. (Color online) (a) Reciprocal space map (RSM) around the neighborhood of the $\{211\}$ reflection for $x=0.2$ film. The vertical and horizontal axes correspond to $\omega$ and $2 \theta$ diffraction angles, respectively, and (b) $2 \theta$ scan around the (211) plane showing splitting of the (211) and (21) peaks suggesting a reduced symmetry.

For this purpose we present data for a film with composition of $x=0.13$. This composition was chosen because it is not only on the FE side but also close to the FE-AFE phase boundary such that we are able to demonstrate that it straddles the FE-AFE phase boundary with minimal external perturbation. Figure 11(a) plots the intensity of a typical $\frac{1}{2}\{h k l\}$ or $\left.\frac{1}{2}(112)\right)$ spot as a function of temperature by laboratory $\mathrm{x}$ ray up to $400{ }^{\circ} \mathrm{C}$. Figure 11 (b) shows that the film exhibits good square hysteresis loops and sharp capacitancevoltage $(C-V)$ peaks (indication of very good $\mathrm{FE}$ behavior) at room temperature. At room temperature the intensity of the spot is negligible. The corresponding systematic changes in the shape of the ferroelectric hysteresis and the $C-V$ loops with increasing temperature are presented in Figs. $11(\mathrm{c})-11(\mathrm{f})$. It is noticed distinctly that at the temperature where the intensity of the spot reaches maximum $\left(\sim 140{ }^{\circ} \mathrm{C}\right)$, the hysteresis loop changes from a square shape to a double hysteresis. It is accompanied by a corresponding change in the $C-V$ curves, where instead of a double hump [as seen in Fig. 11(b)], quadruple humps (two sets of double humps for each sweep direction) are observed. Following this the double hysteresis is found to persist up to highest possible temperature $\left(170{ }^{\circ} \mathrm{C}\right)$. For even higher temperatures, although a double hysteresis was not achievable distinct quadruple humped $C-V$ as well as intensity of the spots persists. In this manner, it is possible to link the appearance of the $\frac{1}{2}\{h k l\}$ spot to the appearance of the macroscopic AFE behavior. 

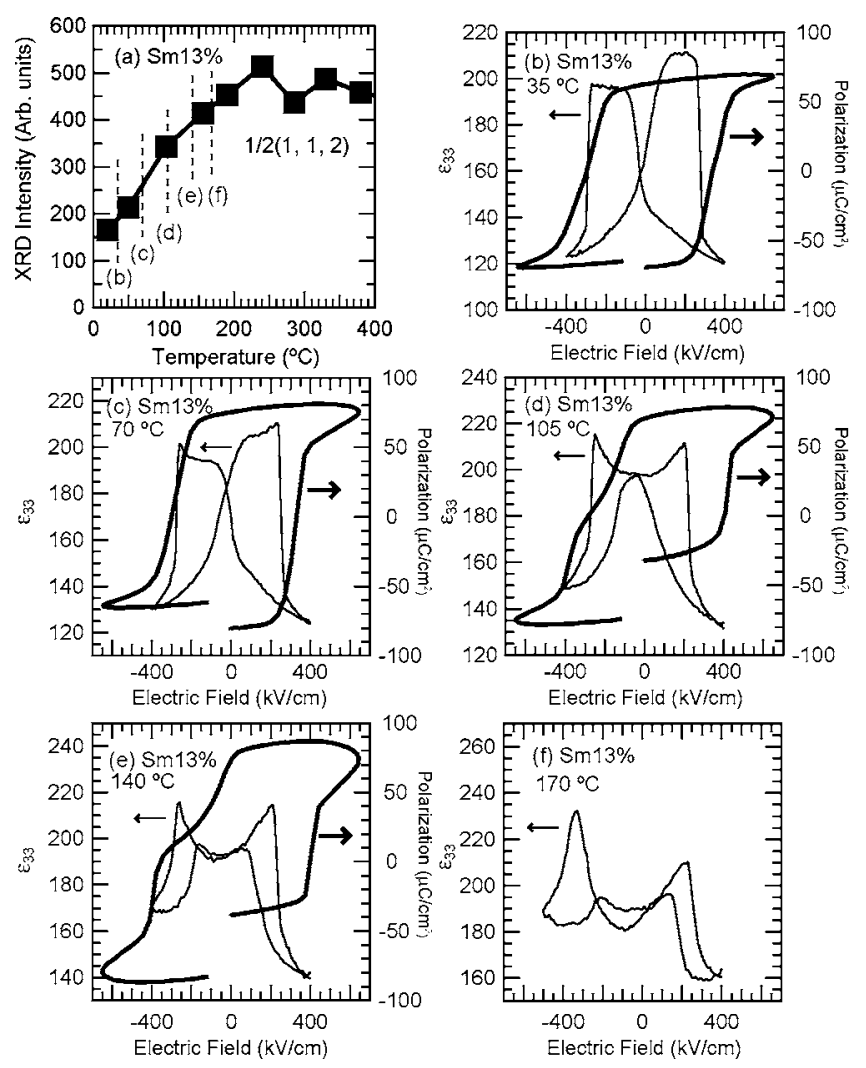

FIG. 11. (a) Change in the x-ray intensity of a $\frac{1}{2}\{h k l\}$ spot as a function of temperature. Capacitance-voltage $(C-V)$ curve and hysteresis loop at (b) $35{ }^{\circ} \mathrm{C}$; (c) $70{ }^{\circ} \mathrm{C}$; (d) $105{ }^{\circ} \mathrm{C}$; (e) $140{ }^{\circ} \mathrm{C}$; and (f) $170{ }^{\circ} \mathrm{C}$ showing FE to AFE transition occurred around $140{ }^{\circ} \mathrm{C}$.

\section{Diffraction studies on $\mathrm{Bi}_{0.7} \mathrm{Sm}_{0.3} \mathrm{FeO}_{3}$ (BSFO with $x=0.3$ )}

At $x=0.3$ [Fig. 12(a)], the [001] ZADP shows only the appearance of fundamental reflections. This indicates that the tilting of the octahedra has disappeared and the system has become more of a cubic aristotype, confirmed by paraelectric behavior in the hysteresis loop as shown in Fig. 12(b). This is attributed to the effect of excessive $\mathrm{Sm}^{3+}$, which has destroyed any polar interactions between the $\mathrm{Bi}^{3+}$ ions, and thus any advantage gained by a change in symmetry order is nullified due to the high concentration of nonpolar dopant cation. ${ }^{55,56}$

\section{PROPOSED MODEL AND CONCLUSIONS}

These detailed diffraction analyses now allow us to describe the local structure evolution across FE-AFE phase transition and postulate the plausible origin for superior electromechanical properties at this phase boundary. The evolution is schematically illustrated in Fig. 13. Fundamentally, this can be linked to two primary dipolar interactions: (i) the already understood long-range (head-to-tail) interactions due to the displacement of the $\mathrm{Bi}^{3+}$ ions that bring $\mathrm{FE}$ order and (ii) the short-range antiparallel (sideways) interactions due to the $\mathrm{Sm}^{3+}$ dopants that induce AFE. In the context of the present BSFO system, occurrence of such interactions can, perhaps, be best viewed as resulting in onset of AFE clusters
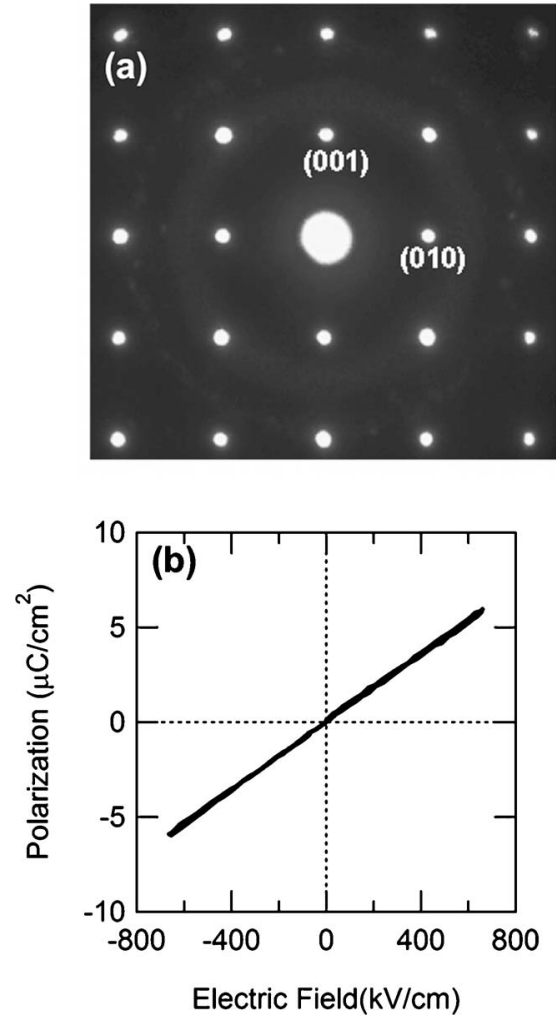

FIG. 12. (a) The [001] ZADP of $x=0.3$ with only fundamental reflections and (b) Polarization hysteresis loops confirms the paraelectric behavior.

where introduction of substituted $\mathrm{Sm}^{3+}$ dramatically modifies the local dipole alignments, although macroscopic FE hysteresis loops may still be observed. Increasing of the $\mathrm{Sm}^{3+}$ concentration leads to concomitant changes in local chemical environment which in turn affects the perovskite unit cell via cation-cation interactions as well as distortions to the oxygen octahedral network. The clusters of AFE regions first undergo nucleation and then grow within a FE matrix and at the critical concentration of $x=0.14$, FE regions are so frustrated

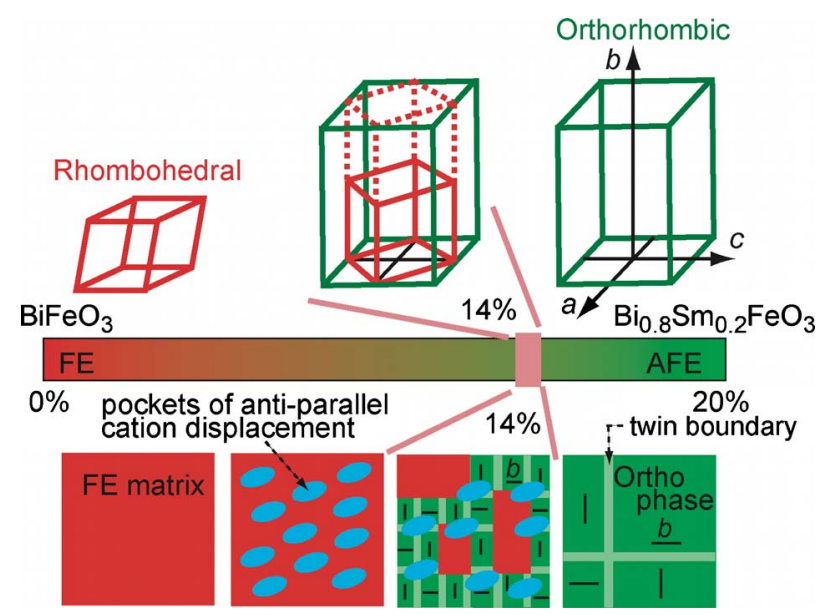

FIG. 13. (Color online) Schematic illustration describing structural evolution across BSFO FE-AFE phase boundary which ultimately link to its functional behavior. 
by the surrounding AFE matrix that it results in an incommensuration phenomenon where the system adopts a complex nanoscale mixture bridging between Bi-rich rhombohedral and Sm-rich orthorhombic phases.

Thus we propose that incommensuration takes place not fortuitously, but as a mechanism with which the BSFO system adapts itself as a compromise between displacement of cations related quadrupling of unit cell for $x<0.14$ and rotation of oxygen-related doubling of unit cell for $x>0.14$ in a manner favoring nanoscale phase co-existence and giving rise to one-variant incommensurate ordering. This implies that incommensurately modulated phase invokes mechanisms involving strong interactions between FE and AFE states, where such competition between the differing polar orders is the key factor that drives the subsequent morphotrophic phase change in this system. This is experimentally seen as a phase transformation into a lower symmetry (triclinic/pseudo-orthorhombic) phase. ${ }^{5}$ accompanied by complex weak reflections in the diffraction patterns. This intricate phase mixture is perceived to be easily perturbed by external fields or stresses, and thus leads to increased electromechanical coefficients. Macroscopic functional AFE behavior is observed only when the $\mathrm{Sm}^{3+}$ concentration increases beyond the critical composition of $x=0.14$. For very large $\mathrm{Sm}^{3+}$ doping concentrations, the AFE disappears and paraelectric phase appears.

Further investigations on the superstructure including the temperature-dependence study ${ }^{4}$ are necessary to understand the precise nature of the octahedral tilt transitions in these new $\mathrm{Sm}$-doped $\mathrm{BiFeO}_{3}$ thin films, and are currently underway. We are also performing electron-energy-loss spectroscopy work on those ordered structures to shed further light on the role of chemical environments in RE-doped BFO superstructures. Similar tilt transitions could be expected for other specific rare-earth dopants which satisfy the critical Goldschmidt factor as discussed above, and these are also being investigated.

In summary, we reported on the structural changes in $\mathrm{BiFeO}_{3}$ as a function $\mathrm{Sm}^{3+}$ doping using comprehensive TEM diffraction analyses, HRTEM, and x-ray diffraction analyses. We show that doping with $\mathrm{Sm}^{3+}$ first creates antiparallel cation displacements in local pockets. With continued additions of $\mathrm{Sm}^{3+}$ a series of phase transitions and superstructures are observed. The specific properties of these structural phases are linked to the evolution of functional properties previously reported in Ref. 5 .

\section{ACKNOWLEDGMENTS}

Research at UNSW was supported by an Australian Research Council (ARC) Discovery Project and a DEST ISL grant. Work at Maryland was supported by access to the Shared Experimental Facilities of the UMD-NSF-MRSEC under Grant No. DMR 0520471, NSF under Grant No. DMR 0603644, and ARO under Grant No. W911NF-07-1-0410. The work was also supported by the W. M. Keck Foundation and NEDO. R.L.W. acknowledges support of the ARC Discovery Program. C.-J. C and V.N. wish to thank C. A. Randall of Penn State University for fruitful discussions on diffraction analysis and technical officers from UNSW and University of Sydney for TEM work. D.K. acknowledges financial support from the Japan Society for Promotion of Science.
${ }^{1}$ R. Guo, L. E. Cross, S.-E. Park, B. Noheda, D. E. Cox, and G. Shirane, Phys. Rev. Lett. 84, 5423 (2000).

${ }^{2}$ Y. Liu, R. L. Withers, and X. Y. Wei, Phys. Rev. B 72, 134104 (2005).

${ }^{3}$ A. Munkholm, S. K. Streiffer, M. V. Ramana Murty, J. A. Eastman, C. Thompson, O. Auciello, L. Thompson, J. F. Moore, and G. B. Stephenson, Phys. Rev. Lett. 88, 016101 (2001).

${ }^{4}$ F. Z. He, B. O. Wells, Z. G. Ban, S. P. Alpay, S. Grenier, S. M. Shapiro, W. D. Si, A. Clark, and X. X. Xi, Phys. Rev. B 70, 235405 (2004).

${ }^{5}$ S. Fujino, M. Murakami, V. Anbusathaiah, S. H. Lim, V. Nagarajan, C. J. Fennie, M. Wuttig, L. Salamanca-Riba, and I. Takeuchi, Appl. Phys. Lett. 92, 202904 (2008).

${ }^{6}$ J. Wang, J. B. Neaton, H. Zheng, V. Nagarajan, S. B. Ogale, B. Liu, D. Viehland, V. Vaithyanathan, D. G. Schlom, U. V. Waghmare, N. A. Spaldin, K. M. Rabe, M. Wuttig, and R. Ramesh, Science 299, 1719 (2003).

${ }^{7}$ Y. H. Chu, Q. Zhan, C. H. Yang, M. P. Cruz, L. W. Martin, T. Zhao, P. Yu, R. Ramesh, P. T. Joseph, I. N. Lin, W. Tian, and D. G. Schlom, Appl. Phys. Lett. 92, 102909 (2008).

${ }^{8}$ G. L. Yuan and S. W. Or, J. Appl. Phys. 100, 024109 (2006).

${ }^{9}$ H. Uchida, R. Ueno, H. Funakubo, and S. Koda, J. Appl. Phys. 100, 014106 (2006).
${ }^{10}$ D. I. Woodward, J. Knudsen, and I. M. Reaney, Phys. Rev. B 72, 104110 (2005).

${ }^{11}$ I. M. Reaney, E. L. Colla, and N. Setter, Jpn. J. Appl. Phys., Part 1 33, 3984 (1994).

${ }^{12}$ D. I. Woodward and I. M. Reaney, Acta Crystallogr. B61, 387 (2005).

${ }^{13}$ Z. K. Xu, X. H. Dai, D. Viehland, D. A. Payne, Z. Li, and Y. D. Jiang, J. Am. Ceram. Soc. 78, 2220 (1995).

${ }^{14}$ Z. Xu, D. Viehland, P. Yang, and D. A. Payne, J. Appl. Phys. 74, 3406 (1993).

${ }^{15}$ Z. Xu, X. H. Dai, and D. Viehland, Appl. Phys. Lett. 65, 3287 (1994).

${ }^{16}$ A. M. Glazer, Acta Crystallogr. B28, 3384 (1972).

${ }^{17}$ A. M. Glazer, Acta Crystallogr. A31, 756 (1975).

${ }^{18}$ L. A. Giannuzzi and F. A. Stevie, Micron 30, 197 (1999).

${ }^{19}$ N. E. Brese and M. Okeeffe, Acta Crystallogr. B47, 192 (1991); I. D. Brown, The Chemical Bond in Inorganic Chemistry: The Bond Valence Model (Oxford University Press, Oxford, New York, 2002).

${ }^{20}$ E. N. Maslen, V. A. Streltsov, and N. Ishizawa, Acta Crystallogr. B52, 406 (1996).

${ }^{21}$ C. Michel, J.-M. Moreau, G. D. Achenbach, R. Gerson, and W. J. James, Solid State Commun. 7, 701 (1969). 
${ }^{22}$ D. I. Woodward, I. M. Reaney, R. E. Eitel, and C. A. Randall, J. Appl. Phys. 94, 3313 (2003).

${ }^{23}$ P. M. Woodward, Acta Crystallogr. B53, 44 (1997).

${ }^{24}$ M. Marezio, J. P. Remeika, and P. D. Dernier, Acta Crystallogr. 26, 2008 (1970).

${ }^{25}$ P. Baettig, C. F. Schelle, R. LeSar, U. V. Waghmare, and N. A. Spaldin, Chem. Mater. 17, 1376 (2005).

${ }^{26}$ E. Sawaguchi, H. Maniwa, and S. Hoshino, Phys. Rev. 83, 1078 (1951).

${ }^{27}$ D. Viehland, Phys. Rev. B 52, 778 (1995).

${ }^{28}$ Y. Cai, F. Phillipp, A. Zimmermann, L. Zhou, F. Aldinger, and A. Ruhle, Acta Mater. 51, 6429 (2003).

${ }^{29}$ X. H. Dai, Z. Xu, and D. Viehland, J. Appl. Phys. 77, 5086 (1995).

${ }^{30}$ I. M. Reaney, A. Glazounov, F. Chu, A. Bell, and N. Setter, Br. Ceram. Trans. 96, 217 (1997).

${ }^{31}$ J. Ricote, D. L. Corker, R. W. Whatmore, S. A. Impey, A. M. Glazer, J. Dec, and K. Roleder, J. Phys.: Condens. Matter 10, 1767 (1998).

${ }^{32}$ A. G. Khachaturyan and D. Viehland, Metall. Mater. Trans. A 38, 2308 (2007).

${ }^{33}$ A. G. Khachaturyan and D. Viehland, Metall. Mater. Trans. A 38, 2317 (2007).

${ }^{34}$ Z. Xu, X. H. Dai, and D. Viehland, Phys. Rev. B 51, 6261 (1995).

${ }^{35}$ D. Viehland, D. Forst, Z. Xu, and J. F. Li, J. Am. Ceram. Soc. 78, 2101 (1995).

${ }^{36}$ D. Forst and D. Viehland, J. Appl. Phys. 76, 5891 (1994).

${ }^{37}$ D. Viehland, X. H. Dai, J. F. Li, and Z. Xu, J. Appl. Phys. 84, 458 (1998).

${ }^{38}$ A. G. Khachaturyan, S. M. Shapiro, and S. Semenovskaya, Phys. Rev. B 43, 10832 (1991).
${ }^{39}$ Z. Xu, X. H. Dai, J. F. Li, and D. Viehland, Appl. Phys. Lett. 71, 2280 (1997).

${ }^{40}$ H. Z. Cummins, Phys. Rep. 185, 211 (1990).

${ }^{41} \mathrm{H}$. He and X. Tan, Phys. Rev. B 72, 024102 (2005).

${ }^{42}$ S. Bhattacharyya, J. R. Jinschek, H. Cao, Y. U. Wang, J. F. Li, and D. Viehland, Appl. Phys. Lett. 92, 142904 (2008).

${ }^{43}$ K. A. Schonau, L. A. Schmitt, M. Knapp, H. Fuess, Rudiger-A. Eichel, H. Kungl, and M. J. Hoffmann, Phys. Rev. B 75, 184117 (2007).

${ }^{44}$ L. A. Schmitt, K. A. Schonau, R. Theissmann, H. Fuess, H. Kungl, and M. J. Hoffmann, J. Appl. Phys. 101, 074107 (2007).

${ }^{45}$ R. Theissmann, L. A. Schmitt, J. Kling, R. Schierholz, K. A. Schonau, H. Fuess, M. Knapp, H. Kungl, and M. J. Hoffmann, J. Appl. Phys. 102, 079902 (2007).

${ }^{46}$ Y. U. Wang, Phys. Rev. B 74, 104109 (2006).

${ }^{47}$ T. Asada and Y. Koyama, Phys. Rev. B 75, 214111 (2007).

${ }^{48}$ B. Noheda, L. Wu, and Y. Zhu, Phys. Rev. B 66, 060103(R) (2002).

${ }^{49}$ G. Shirane and S. Hoshino, J. Phys. Soc. Jpn. 6, 265 (1951).

${ }^{50}$ N. Setter and L. E. Cross, J. Appl. Phys. 51, 4356 (1980).

${ }^{51}$ R. L. Withers, Y. Liu, P. Woodward, and Y. I. Kim, Appl. Phys. Lett. 92, 102907 (2008).

${ }^{52}$ H. Zheng, I. M. Reaney, W. E. Lee, N. Jones, and H. Thomas, J. Am. Ceram. Soc. 85, 2337 (2002).

${ }^{53}$ E. L. Colla, I. M. Reaney, and N. Setter, J. Appl. Phys. 74, 3414 (1993).

${ }^{54}$ X. Qi, M. Wei, Y. Lin, Q. Jia, D. Zhi, J. Dho, M. G. Blamire, and J. L. MacManus-Driscoll, Appl. Phys. Lett. 86, 071913 (2005).

${ }^{55}$ I. Grinberg, M. R. Suchomel, P. K. Davies, and A. M. Rappe, J. Appl. Phys. 98, 094111 (2005).

${ }^{56}$ M. R. Suchomel and P. K. Davies, J. Appl. Phys. 96, 4405 (2004). 